\section{Introducción: ¿Por qué un monográfico sobre las técnicas construtivas?}

JuAn ANTONIO QUiRós CASTILLO

\section{Resumen}

En esta introducción se expone la importancia que ha tenido en los últimos años el estudio de las técnicas constructivas en el marco de la construcción disciplinar de las técnicas de construcción.

Asimismo se plantean los vectores que han guiado la recopilación de artículos que conforman este monográfico.

Palabras clave: Técnicas constructivas, Arqueología de la Arquitectura
El número 4 de la revista Arqueología de la Arquitectura está dedicado de forma monográfica al estudio de las técnicas constructivas medievales, dedicando una atención especial a las transformaciones que han tenido lugar en las formas de construir en varios sectores europeos en los siglos centrales de la Edad Media.

$\mathrm{Si}$ bien es cierto que en una fase inicial el desarrollo de determinados instrumentos de análisis (en primer lugar la estratigrafía de paramentos) o de contextos de actuación (la intervención patrimonial) han tenido un peso muy importante en la definición y en la construcción de una $\mathrm{Ar}$ queología de la Arquitectura, desde hace ya varios años se han ido incorporando nuevas temáticas que están contribuyendo a ampliar sus contenidos.

Una de las más importantes y fecundas líneas de investigación que ha desarrollado la Arqueología de la Arquitectura en los últimos años ha sido el análisis de las técnicas constructivas desde ópticas muy distintas (históricas, tecnológicas, estéticas, etc.). Las razones de este interés son muy variadas, ya que se ha comprendido que a través del conocimiento de las formas de construir era posible realizar investigaciones tanto de carácter básico como aplicado en el campo del patrimonio edificado.

Por un lado, la discontinuidad tecnológica que ha provocado la industrialización contemporánea ha comportado la desaparición de un notable número de formas de construir, lo que genera importantes problemas a la hora de intervenir sobre el patrimonio edificado histórico. Asimismo, las técnicas constructivas han constituido un criterio básico de análisis histórico de las construcciones en términos de atribución cronológica, cultural o estilísticas. Pero además, los arqueólogos han mostrado que, a través del análisis y la reconstrucción de las formas de construir, y de la articulación del artesanado es posible acercarse de forma más rigurosa a la historia social de la arquitectura.

Teniendo en cuenta estos intereses, desde la redacción de la revista Arqueología de la Arquitectura se ha pretendido reunir una serie de textos relativos a esta temática, teniendo en cuenta la notable heterogeneidad que caracteriza las distintas escuelas que operan en el ámbito de la «Arqueología de la Arquitectura».

Precisamente el estudio de las técnicas de construcción ha constituido uno de los primeros intereses de los arqueólogos. Ya desde el siglo XV fue objeto de estudio las formas de construir de los romanos a partir de las ruinas. Este interés, que adquirió un notable desarrollo durante el período renacentista, inspiró la realización de obras paradigmáticas. Basta pensar la influencia que en las obras de Brunelleschi, por citar solamente un autor, tuvo la arquitectura romana. 
Pero un estudio de carácter científico sobre las técnicas antiguas se puede rastrear a partir del Iluminismo, cuando se realizan tratados, estudios e investigaciones sobre las técnicas de construir de los antiguos, e incluso se plantea el problema de la datación de las técnicas constructivas.

No obstante, será a partir de los siglos XIX y XX cuando se desarrollen estudios de un cierto calado sobre las técnicas de construcción antiguas, de tal manera que en las síntesis de historia de la arquitectura de finales del XIX e inicios del XX, como las de Durm (1905) o de Choise (1873), se presta una atención especifica a los aspectos técnicos, llegando incluso a privilegiarlos por encima de los estéticos.

Ya en el siglo XX se desarrollará una corriente de estudios, que ha sido definida como "estilístico-comparativa" (CAGNANA 1994: 39), basadas en presupuestos de naturaleza empírica y positivista, en cuanto que pretende realizar una clasificación sistemática de las técnicas constructivas de monumentos de distintos períodos históricos. Los trabajos de autores como Van Deman (1912), Blake (1947) o Lugli (1957) son indudablemente los más importantes de esta corriente de estudios. Las aportaciones de estos investigadores han sido fundamentales para el conocimiento riguroso de la arquitectura romana, aunque su principal limitación ha residido en el marco teórico en el que llevaron a cabo este tipo de investigaciones.

En un período caracterizado por la influencia de idealismo, se consideró la historia de la arquitectura y de las técnicas constructivas romanas como una historia que evolucionaba hacia formas constructivas cada vez más perfectas desde el período republicano al imperial, para luego decaer de forma paralela a la crisis del imperio. Dotando de valores formales a los paramentos y a los distintos opus con los que se clasificaban las formas de construir, se dejaron de lado obras consideradas «menores» en términos estéticos, pero esenciales para comprender las complejas dinámicas de la arquitectura romana. Por otro lado, esta aproximación idealista impedía observar un fenómeno complejo, pero muy frecuente, representado por la coexistencia en un mismo territorio de técnicas constructivas muy distintas.

Con todo, los modelos y las clasificaciones tipocronológicas construidos por estos autores, que llegaron a alcanzar una gran solidez y precisión con autores como Lugli, se hicieron demasiado rígidos, de tal manera que las divergencias que empezó a plantear a mediados de siglo las excavaciones estratigráficas generó un agrio conflicto entre las culturas tipológicas y estratigráficas. El desencuentro entre Lamboglia y el propio Lugli ilustra perfectamente este conflicto a la hora de analizar las técnicas de construcción y, en general, la datación de la arquitectura romana.
Solamente a partir de los años 70 se ha asistido a una profunda renovación teórica y metodológica en el estudio arqueológico de las arquitecturas, a costa de la ruptura entre la tradición artística-formal que había dominado la arqueología de los dos primeros tercios del siglo XX y una tradición que se reconocía en nuevos modelos teóricos y nuevas formas de análisis de la arquitectura. El desarrollo de aproximaciones de carácter tecnológico y social ha contribuido de forma decisiva a reformular sobre nuevas bases este tipo de estudio (MANNONi 1997; Brogiolo 1996; CaGnana 2000).

Así por ejemplo el estudio de las técnicas constructivas romanas ha sufrido en este contexto una profunda renovación (TORELLI 1980; CARANDINI 1988, BESSAC 1991, etc), sustituyendo la aproximación estético-formal por una visión tecnológica y social.

Asimismo la ampliación cronológica de la práctica arqueológica hacia otros períodos más recientes ha tenido como consecuencia la realización de nuevos estudios sobre las técnicas constructivas de época medieval y postmedieval (Kimpel 1977; Mannoni 1994). De hecho, la edición de trabajos recientes dedicados al estudio de las técnicas constructivas medievales en territorios específicos (FIORANI 1996; SÁNCHEZ ZUFIARRE 2006), o en términos generales (CASSANELLI 1995), demuestra la importancia y la incidencia de este tipo de estudios en las investigaciones más recientes sobre la arquitectura medieval.

Con el fin de ofrecer una perspectiva amplia del estado actual de las investigaciones realizadas en torno a esta temática, se han reunido en el presente volumen los trabajos de una docena de autores que, desde planteamientos teóricos y metodológicos distintos, abordan el estudio arqueológico de construcciones de época medieval. Los autores invitados a la redacción de los distintos capítulos, son investigadores reconocidos y de prestigio pertenecientes a varias instituciones científicas italianas, españolas y francesas. La organización de los distintos capítulos es de carácter geográfico y temático, mientras que se ha incluido un trabajo inicial realizado por T. Mannoni que tratará de forma amplia y genérica los problemas relativos al análisis arqueológico de las técnicas constructivas.

La inevitable heterogeneidad de estos estudios, favorecida por la diversidad de territorios y de tipologías constructivas analizadas, ha sido considerada en esta ocasión como un aspecto muy positivo y necesario para lograr tener una visión amplia de cómo la arqueología aborda el estudio tecnológico de la arquitectura. De hecho, en cada uno de los trabajos se ha pretendido exponer explorar los planteamientos epistemológicos, conceptuales e instrumentales que utilizan los distintos grupos que trabajan en el ámbito de la Arqueología de la Arquitectura. 
Asimismo se ha querido analizar de forma comparada experiencias realizadas en varios sectores de Europa Occidental (Italia, Francia, España), ofreciendo síntesis y balances críticos sobre los estudios realizados y el estado actual de los conocimientos sobre las técnicas constructivas en época medieval. De forma excepcional se han incluido dos estudios relativos al Mediterráneo Oriental, que servirán como punto de comparación con la producción realizada en nuestro entorno más inmediato.

Por último se ha querido prestar una especial atención al problema de la aparición de las técnicas constructivas complejas en torno al año mil, vinculando la transformación de las técnicas constructivas y la organización del artesanado a los cambios sociales que ha supuesto la feudalización de la sociedad.

Teniendo en cuenta el papel que el estudio de las técnicas de construcción tiene en la actualidad en la construcción de la arqueología de la arquitectura, se piensa que los trabajos recogidos en este volumen han de contribuir a alargar el debate teórico e interpretativo de la arquitectura, superando de esta manera la «dictadura» de la estratigrafía que parece caracterizar los trabajos realizados en el ámbito de la Arqueología de la Arquitectura.

\section{Bibliografía}

BESSAC J. C., 1986, L'outillage traditionnel du tailleur de pierre de l'antiquité à nos jours, París.

BROGIOLO G.P., 1996, Prospettive per l'archeologia dell'architettura, $A r-$ cheologia dell'architettura I, pp. 11-15

CAgnana A. 1994, Archeologia della produzione fra tardo-antico e Altomedioevo. Le tecniche murarie e l'organizzazione dei cantieri, en Atti del $4 .^{\circ}$ Seminario sul tardoantico e l'altomedioevo in Italia centrosettentrionale, (Monte Barro-Galbiate, Lecco, 2-4 settembre 1993), Mantova, pp. 39-52.

Cagnana A., 2000, Archeologia dei materiali da costruzione, Mantova

CARANDInI A., 1988, Schiavi in Italia. Gli strumenti pensanti dei Romani fra tarda Repubblica e medio Impero, Roma.

Cassanelli R. (ed.), 1995, Talleres de arquitectura en la Edad Media, Barcelona.

FIORANI D., 1996, Tecniche costruttive murarie medievali: il Lazio meridionale, Roma.

KIMPel D., 1977, Le développement et la taille en série dans l'architecture médiévale et son rôle dans l'histoire économique, Bulletin Monumental 177, pp. 195-222.

Mannoni T. 1994, Caratteri costruttivi dell'edilizia storica, Genova

MANNONI T., 1997, Il problema complesso delle murature storiche in pietra 1. Cultura materiale e cronotipologia, Archeologia dell'Architettu$r a$ II, pp. 15-24.

TORELl M., 1980, Innovazioni nelle tecniche edilizie romane tra il I sec. a. C. e il I sec. d.C, en Tecnologia, economia e società nel mondo romano, Como, pp. 139-162. 